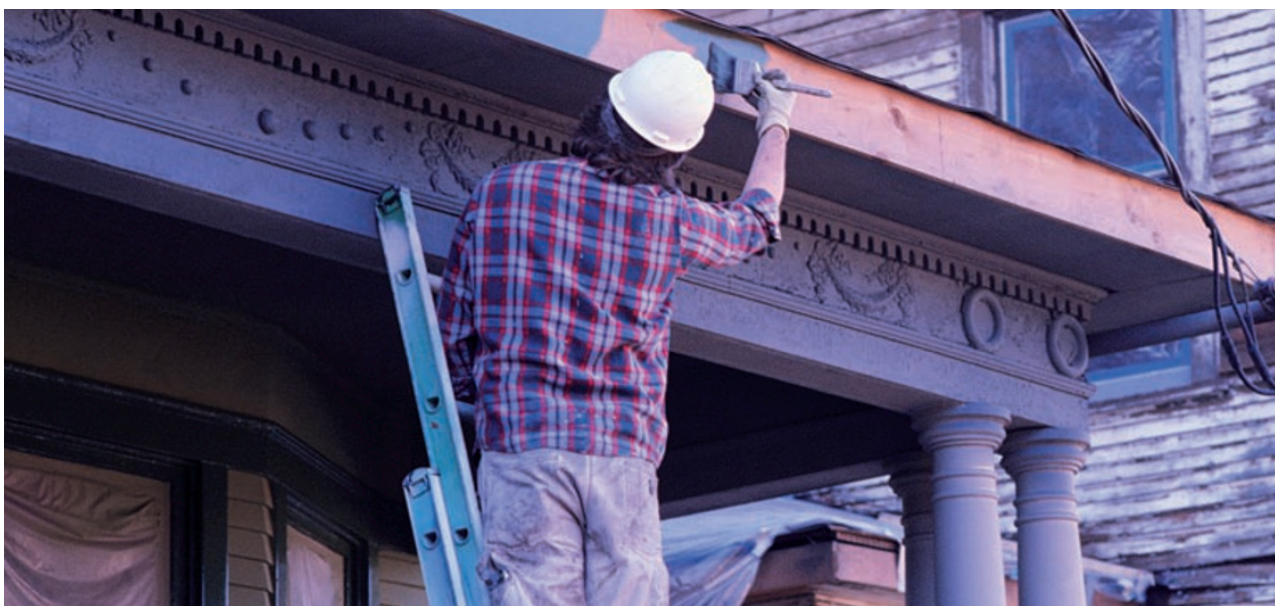

TECHNOLOGY

\section{New front-end for high-throughput resequencing}

Recent years have seen remarkable innovation in sequencing technologies, making them highthroughput, faster and more cost effective. However, isolation of the target DNA to be sequenced has remained a bottleneck. Three new reports in Nature Methods provide an alternative to the traditional PCR- and BAC-based approaches to this step. Two of them describe microarraybased enrichment of target DNA, whereas the third involves targeted multiplex amplifications, all of which are ideally suited to large-scale, multiplex and cost-effective genome resequencing.

Okou et al. and Albert et al. have devised similar methods in which genomic DNA is fragmented, repaired by addition of adapters and captured on custom-made high-density microarrays. Following elution and amplification, this target pool is ready for resequencing. Both methods prove to be highly accurate - the method of Albert et al. captured 6,700 exons, adding up to $5 \mathrm{Mb}$, within which it identified 94-97\% of variants, whereas Okou et al. achieved $>99 \%$ identification in a contiguous $300-\mathrm{kb}$ region on the $\mathrm{X}$ chromosome.

The approach of Porreca and Zhang et al. is quite different. In the first step, the authors synthesized a library of targeting oligonucleotide precursors directly on a microarray. Upon release from the array, the oligonucleotide precursors were PCR amplified and digested to create single-standed 'capture probes', the ends of which were designed to hybridize on either side of the target sequence. The 'capture' itself involves polymerase-driven gap filling and ligation to create circular DNA molecules that are then enriched, amplified and can be turned into a shotgun sequencing library.
This multiplexing approach is highly specific ( $98 \%$ of mapped reads corresponding to targeted regions) and can capture $\sim 10,000$ targets in one reaction, raising the possibility that all annotated protein-coding human sequences could be queried in just a few reactions. However, the resequencing data at heterozygous positions are skewed away from the expected 0.5 ratio, something that warrants further investigation but might be due to inefficient hybridization to genomic DNA. Improving the uniformity of target capture and amplification is another important focus for future improvement - the $\sim 10,000$ targets that were captured represent $20 \%$ of the total targeted sequence.

All three approaches bring important advances over the exisiting 'front-end' methods, in terms of throughput and cost. They are also free of the sequence-related constraints that plague restriction-enzyme-based approaches. Further improvements in these 'genome partioning' methods are undoubtedly just around the corner and, with them, costeffective, high-throughput, targeted genomic resequencing.

Magdalena Skipper

ORIGINAL RESEARCH PAPERS Okou, D. T. et al. Microarraybased genomic selection for high-throughput resequencing. Albert, T. J. et al. Direct sequencing of human genomic loci by microarray hybridization. Nature Methods 14 October 2007 (doi:10.1038/nmeth1111)| Porreca, G. J. \& Zhang, K. et al. Multiplex amplification of large sets of human exons. Nature Methods 14 October 2007 (doi:10.1038/nmeth1110) FURTHER READING Shendure, J. et al. Advanced sequencing technologies: methods and goals. Nature Rev. Genet. 5, 335-344 (2004) | Hoheisel, J. D. Microarray technology: beyond transcript profiling and genotype analysis. Nature Rev. Genet. 7, 200-210 (2006) Nature Methods 14 October 2007 (doi:10.1038/nmeth1109)|

\section{In the news}

\section{INTERFERING WITH CONCEPTION}

Some 60 years after the Pill was introduced, modern birth control is set to get a much-needed facelift the development of a revolutionary, no-risk contraceptive method has been announced.

The new approach — developed by the researchers at Brigham and Women's Hospital of Harvard Medical School in Boston, USA has been referred to as "genetic contraception. In fact, it could one day become the first of an 'entirely new class of contraceptives', leading researcher Dr Zev Williams hopes" (ABC News, 19 October 2007). The contraceptive uses RNAi against "a gene that produces a protein called ZP3. The protein, which is part of the coating that surrounds a woman's egg, enables sperm to bind to the outer layer" (The Daily Telegraph, 20 October 2007).

Unlike the existing birth control drugs, this approach is nonhormonal and does not control the woman's monthly cycle. "Instead it would allow ovulation to occur as normal but would prevent the sperm penetrating the egg..." (The Independent, 17 October 2007). Another important aspect of this approach is that ZP3 “doesn't occur anywhere else in the body, so the effect is highly targeted" (The Independent, 17 October 2007).

So far, the Williams-led team have tested their method in mice, and the human trials are unlikely to begin sooner than within 10 years. Besides, more work is needed "to find a way of delivering the drug effectively [...]. In humans, it is likely to be given via a skin patch or a vaginal suppository" (The Times, 17 October 2007).

Although hailed as being safe, "there is no guarantee that sideeffects could be avoided completely" (BBC News, 17 October 2007). Magdalena Skipper

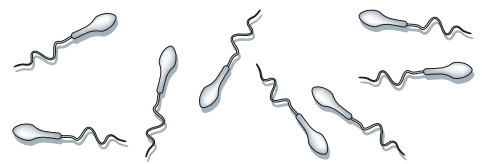

